\title{
The role of surface defects in laser-induced thermal desorption from metal surfaces
}

\author{
J. Viereck ${ }^{\text {a }}$, F. Stietz ${ }^{\text {a }}$, M. Stuke ${ }^{\text {b }}$, T. Wenzel ${ }^{\text {a }}$, F. Träger ${ }^{\text {a.* }}$ \\ a Fachbereich Physik, Universität Kassel, Heinrich-Plett-Strasse 40, D-34132 Kassel, Germany. \\ ${ }^{\mathrm{b}}$ Max-Planck-Institut für Biophysikalische Chemie, P.O. Box 2841, D-37018 Göttingen. Germamy
}

Received 18 February 1997; accepted for publication 3 March 1997

\begin{abstract}
Laser-induced thermal desorption of Na dimers from rough sodium surfaces adsorbed on quartz substrates has been studied. For this purpose, laser pulses with $\lambda=532$ and $1064 \mathrm{~nm}$ and $7 \mathrm{~ns}$ duration were used. The kinetic energy distribution and the integral desorption signal of the dimers were determined as a function of the laser fluence. Measurements were also performed after reducing the surface roughness by annealing at different temperatures. The fluence dependence of the integral desorption rate exhibits a plateau which follows and precedes a sharp increase. The results indicate that the Na dimers come off preferentially from special sites of low binding energy and low coordination number. Two of these sites can be distinguished. They exhibit different annealing behavior, different binding energies and can be depleted selectively by choosing the laser fluence appropriately. $\mathrm{O} 1997$ Elsevier Science B.V.
\end{abstract}

Keywords: Desorption induced by electronic transitions: Photon stimulated desorption; Surface structure. morphology, roughness, and topography; Thermal desorption

A detailed understanding of laser-induced desorption and ablation is not only of scientific interest, but opens up the possibility of exploiting such reactions for a large number of applications. Besides the desorption of molecular species from many different surfaces, the removal of substrate atoms from semiconductors [1,2], metals [3-9] and insulators [10] by laser radiation has been studied. In order to investigate the underlying desorption mechanisms, measurements of the desorption rate and the kinetic energy distributions of the ejected atoms as a function of laser wave-

\footnotetext{
* Corresponding author. Fax: +495618044518;
} e-mail: traeger@physik.uni-kassel.de length, angle of incidence, laser fluence and substrate temperature have been performed. It turns out that metal atoms like $\mathrm{Au}, \mathrm{Ag}, \mathrm{Al} . \mathrm{K}$ or $\mathrm{Na}$ can even desorb in a non-thermal reaction [3 9], thermal evaporation only coming into effect for high fluences $[9,11]$. In addition to atoms, dimers can be removed from metal or semiconductor surfaces under irradiation with short laser pulses [11-14]. For example, Viereck et al. [12] have found that $\mathrm{Na}$ dimers can desorb from rough $\mathrm{Na}$ surfaces and that the mechanism is non-thermal. Under appropriately chosen conditions, the fraction of detached $\mathrm{Na}$ dimers can even surmount the fraction of desorbed $\mathrm{Na}$ atoms [11].

The studies carried out so far have given strong evidence that non-thermal desorption is based on 
local electronic excitations at the surface, and can be explained in the framework of the well-known scenario of Menzel, Gomer and Redhead [15, 16]. Atoms and dimers desorb predominately from binding sites of the metal surface which have low coordination numbers. These sites can be regarded as defects, and exhibit specific electronic properties which differ from those of extended, low-index single-crystal surfaces (see, e.g., Refs. [1,2,9,12]). As a consequence, the appropriate choice of the applied laser wavelength makes possible the selective depletion of certain sites by laser-induced nonthermal desorption [9].

Other experiments have shown that defects also play an essential role in thermal desorption $[1,17,18]$. For example, Dickinson et al. [17] studied the role of defects on the emission of neutral atoms and molecules from $\mathrm{NaNO}_{3}$. They intentionally increased the number of these sites of low coordination number by electron bombardment, and found that new absorption centers were produced due to defect creation. As a consequence, a larger temperature rise is obtained for a given laser fluence. Thus, thermally activated rate-limiting steps are "catalytically" enhanced by producing defects. Similar observations were made for the laser-induced desorption of $\mathrm{MgO}$ [18]. Another example where surface defects play an essential role in promoting thermal desorption is the removal of Fe atoms from iron surfaces [19].

In a continuation of our earlier work, the present paper reports experiments with the objective of further clarifying the role of surface defects in laser-induced thermal desorption. For this purpose a rather peculiar process at first glance (i.e. the desorption of $\mathrm{Na}$ dimers) constitutes a particularly interesting case, since the rate of desorbed $\mathrm{Na}_{2}$ can be about four times as large as the rate of atoms removed simultaneously $[9,12]$. In contrast, a fraction of only $10 \%$ of the desorbed material consists of dimers in a classical thermal desorption experiment (TPD). Obviously, defects play different roles depending on the heating rate, which can be as high as $10^{9} \mathrm{~K} \mathrm{~s}^{-1}$ under laser irradiation, but typically amounts to as little as several $\mathrm{K} \mathrm{s}^{-1}$ in TPD. In the experiments described here, rough $\mathrm{Na}$ surfaces have been prepared by the deposition of $\mathrm{Na}$ atoms on a dielectric substrate held at cryogenic temperature. The deposited atoms form small particles, the surfaces of which offers a considerable and reproducible number of defects. This not only guarantees large desorption signals [3], but makes it possible to examine whether thermal desorption with pulsed laser light can be controlled such that different kinds of binding sites with low coordination number can be depleted selectively. For the unambiguous interpretation of the measurements described below, deliberate variation of the surface roughness by heat treatment turns out to be essential.

The experimental arrangement has been described in detail elsewhere [9], and basically consists of an ultrahigh vacuum system with the sample, two lasers for stimulating desorption and photoionizing the desorption products, and a timeof-flight mass spectrometer. A thermal beam of $\mathrm{Na}$ atoms with well-defined constant flux is directed onto the quartz substrate in order to deposit a predetermined coverage of atoms. After preparation at a temperature of $80 \mathrm{~K}$, the sample is irradiated with p-polarized light from a Nd:YAG laser at $\lambda=532$ or $1064 \mathrm{~nm}$ in order to stimulate desorption. The angle of incidence is $50^{\circ}$ and the pulse duration $7 \mathrm{~ns}$ at a repetition rate of $9 \mathrm{~Hz}$. At a distance of $21 \mathrm{~mm}$ in front of the substrate, the $\mathrm{Na}$ dimers detached from the metal surface are ionized with the light of an excimer laser operating at $\lambda=248 \mathrm{~nm}$. The corresponding photon energy of $5.0 \mathrm{eV}$ lies only a little above the ionization threshold of $\mathrm{Na}_{2}$ [20]. Therefore, "soft" ionization of the desorbed dimers is accomplished and there is only very little dissociation into monomers [12]. On the other hand, the photon energy used is too low for single-photon ionization of detached $\mathrm{Na}$ atoms. The $\mathrm{Na}_{2}^{+}$ions generated pass the time-offlight mass spectrometer and are detected with a secondary electron multiplier. The ion signal is processed by a boxcar integrator and stored on a computer. By varying the delay time between the two laser pulses used for desorption and ionization, the time-of-flight distributions of the desorbed $\mathrm{Na}$ dimers are determined and subsequently converted into kinetic energy distributions [21]. The choice of $\mathrm{Na}$ as the material for the measurements described here is motivated by two arguments. Firstly, the dimers can be ionized with a single 
laser photon, and secondly, the $\mathrm{Na}$ coverage can be evaporated entirely from the substrate at a temperature of only $\sim 400 \mathrm{~K}$ after completion of a measurement. This makes it possible to use the same substrate repeatedly and ensures reproducible preparation of the sample as well as reproducible desorption signals.

As an example, Fig. 1 displays a time-of-flight spectrum of $\mathrm{Na}$ dimers desorbed with p-polarized laser light of $i=532 \mathrm{~nm}$. The $\mathrm{Na}$ coverage was $1.1 \times 10^{16}$ atoms $\mathrm{cm}^{-2}$, which corresponds to an average particle size of $R_{\mathrm{av}}=21 \mathrm{~nm}$ [22]. The fluence of the Nd:YAG laser was set to a value of $\phi=7.5 \mathrm{~mJ} \mathrm{~cm}^{-2}$. If the fluence is increased, the maximum of the time-of-flight spectrum is shifted

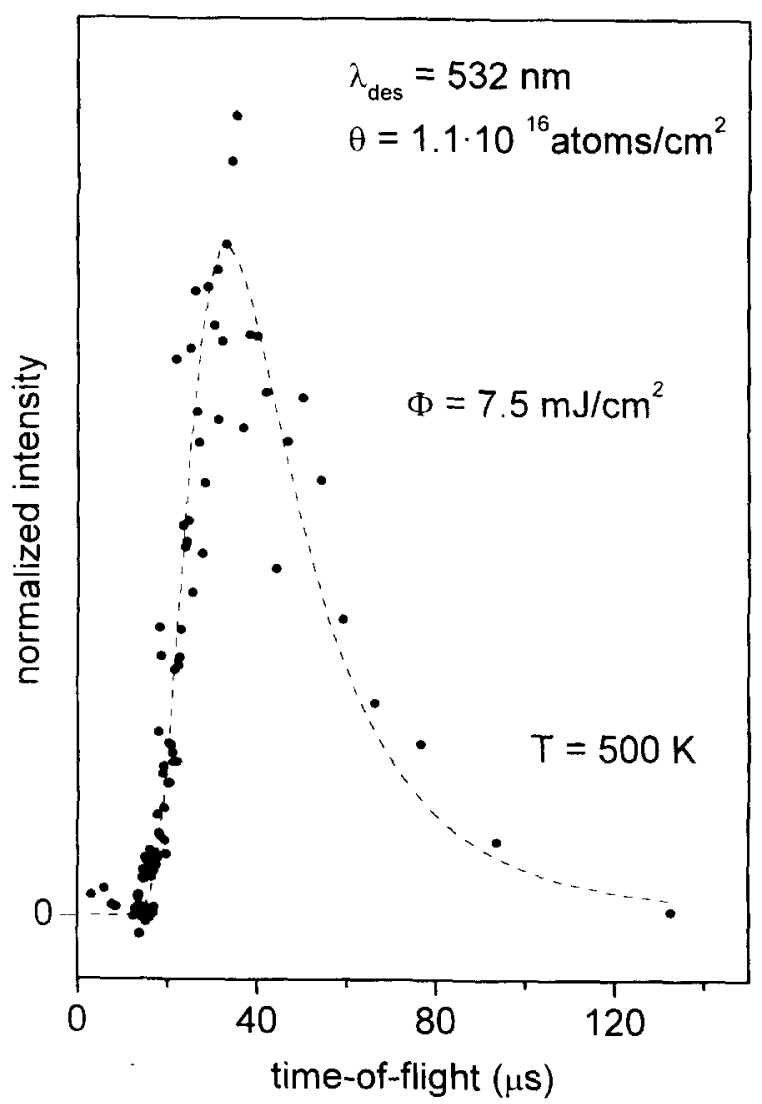

Fig. 1. Time-of-flight spectrum of $\mathrm{Na}$ dimers desorbed with laser light of wavelength $\hat{\lambda}=532 \mathrm{~nm}$. The total sodium coverage was $1.1 \times 10^{16}$ atoms $\mathrm{cm}^{-2}$ which corresponds to an average particle size of $21 \mathrm{~nm}$. The fluence of the Nd:YAG laser was set to a value of $7.5 \mathrm{~mJ} \mathrm{~cm}{ }^{-2}$. The solid line corresponds to a fit by Eq. (1) (see text for details). to shorter flight times. The shape of the distribution, however, does not change noticeably if the fluence remains below about $\phi=25 \mathrm{~mJ} \mathrm{~cm}^{-2}$. The solid line in Fig. 1 is a theoretical curve which was fitted to the experimental data and will be explained below.

Fig. 2 displays the total number of sodium dimers desorbed in one experimental run with p-polarized laser light of $\lambda=532 \mathrm{~nm}$ (top) and $\lambda=$ $1064 \mathrm{~nm}$ (bottom). These data were obtained by measuring the time-of-flight distribution as explained above, with 1000 laser pulses of constant fluence. Then, the integral desorption rate was computed to give one data point of Fig. 2. Subsequently, the sample was freshly prepared and the procedure was repeated for a new value of the fluence, and so forth. As can be seen from Fig. 2a,
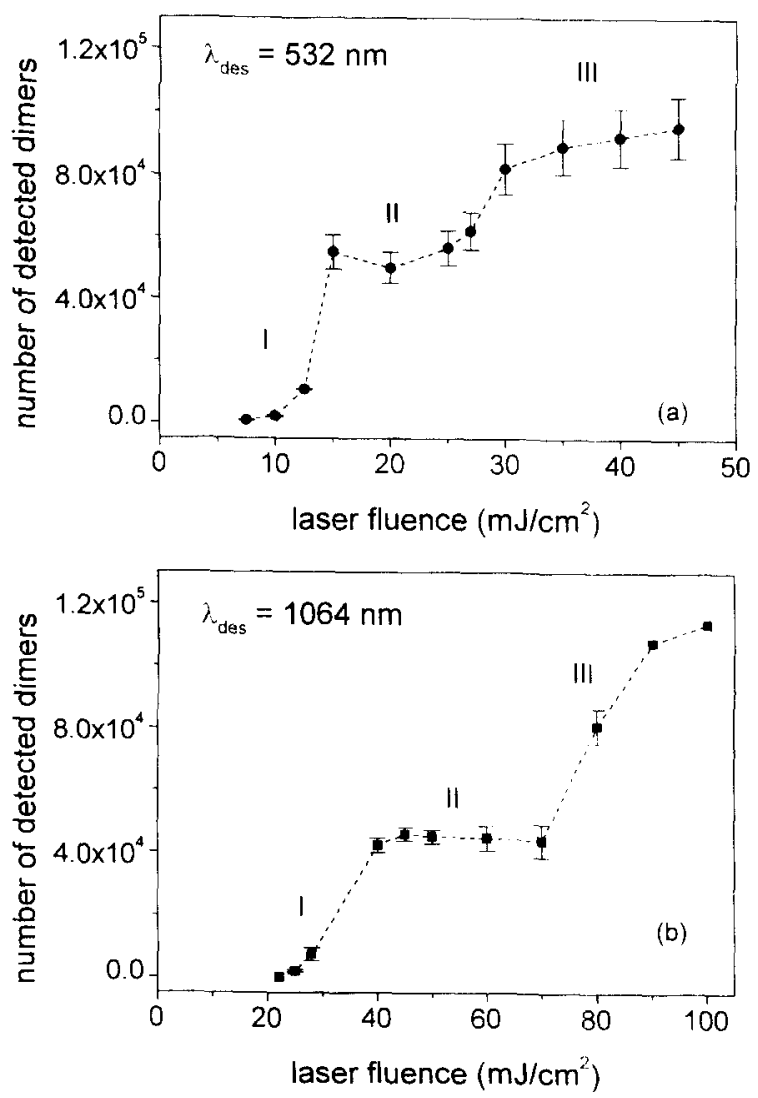

Fig. 2. Number of detected $\mathrm{Na}$ dimers desorbed with laser light of wavelength $\lambda=532 \mathrm{~nm}$ (a) and $1064 \mathrm{~nm}$ (b) as a function of laser fluence. The coverage was $1.1 \times 10^{16}$ atoms $\mathrm{cm}^{-2}$. 
the number of desorbed dimers first rises sharply as a function of laser fluence for $\lambda=532 \mathrm{~nm}$ and reaches a plateau ranging from approximately $\phi=$ 15 to $25 \mathrm{~mJ} \mathrm{~cm}^{-2}$. Further enhancement of the fluence results in a second increase in the number of desorbed dimers. For $\lambda=1064 \mathrm{~nm}$, a very similar behavior is observed (Fig. 2b). The dependence of the number of desorbed dimers, however, is shifted by about $20 \mathrm{~mJ} \mathrm{~cm}^{-2}$ to larger values of the fluence. This reflects the weaker absorption of laser light with $\lambda=1064 \mathrm{~nm}$ making desorption less efficient as compared to $\lambda=532 \mathrm{~nm}$. For the discussion of the data in the rest of this paper, it will be useful to divide the fluence dependence of Fig. 2 into three regimes denoted as I, II and III. The number of desorbed dimers rises in regimes I and III but remains constant in regime II. The integral desorption signal in regime II around $\phi=20 \mathrm{~mJ} \mathrm{~cm}^{-2}$ and $\phi=40 \mathrm{~mJ} \mathrm{~cm} \mathrm{~cm}^{-2}$ for $\lambda=532$ and $\lambda=1064 \mathrm{~nm}$, respectively, is approximately identical. This means that the same total number of dimers is detached independent of the wavelength of the incident laser light.

Fig. 3 illustrates the influence of heat treatment on the number of desorbed dimers. The data were taken as follows. Sodium was deposited on the substrate at $80 \mathrm{~K}$ as outlined above. Subsequently, the sample was annealed for a certain period of time at $T_{\text {ann }}>80 \mathrm{~K}$ and then cooled to the initial temperature of $80 \mathrm{~K}$ at which the time-of-flight

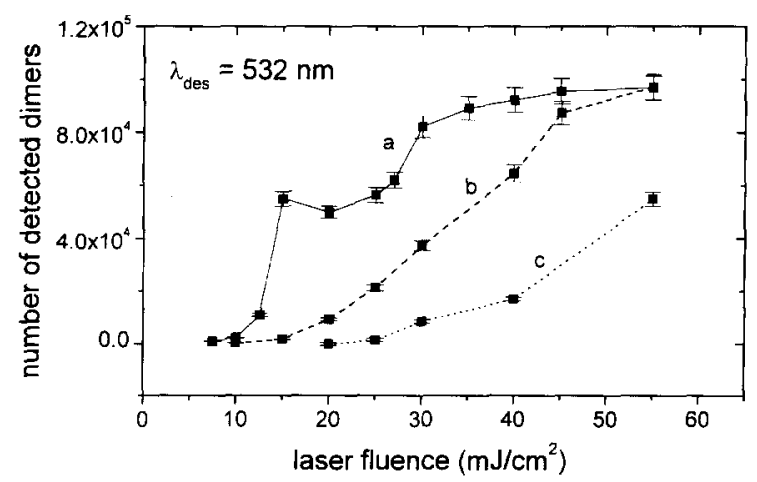

Fig. 3. Number of detected $\mathrm{Na}$ dimers desorbed with laser light of wavelength $\lambda=532 \mathrm{~nm}$. Trace a was obtained without annealing of the surface (same measurement as in Fig. 2a), trace b with annealing at $140 \mathrm{~K}$ for $5 \mathrm{~min}$ and trace $c$ with annealing at $200 \mathrm{~K}$ for $30 \mathrm{~min}$. spectra were recorded. Fig. 3 displays the integral desorption signal as a function of laser fluence without annealing of the sample (trace a, same measurement as shown in Fig. 2a) and with annealing at $140 \mathrm{~K}$ for $5 \mathrm{~min}$ (trace b) and at $200 \mathrm{~K}$ for $30 \mathrm{~min}$ (trace $\mathrm{c}$ ). The wavelength used for desorption was $\lambda=532 \mathrm{~nm}$. For low fluence values the number of dimers which can be desorbed from the annealed surface is substantially smaller as compared to the sample surface initially prepared at low temperature. In particular, the increase in the number of desorbed dimers between 10 and $15 \mathrm{~mJ} \mathrm{~cm}{ }^{-2}$ and the subsequent plateau do longer appear after annealing. Instead, an exponential increase is observed which sets in at a larger fluence if annealing is accomplished at $200 \mathrm{~K}$ as compared to $140 \mathrm{~K}$.

We first conclude from the data that the desorption of sodium dimers obviously occurs as a thermal process for the fluence range and wavelengths used in the present study. This is supported by the shift of the maximum of the time-of-flight spectrum with increasing laser fluence. Furthermore, the experimental data can be fitted by a Maxwellian which describes the time-of-flight spectrum quite well for low fluences (solid line, Fig. 1). Since the desorption signal generated by pulsed laser ionization measures the density of the desorbed species, this Maxwellian reads as follows [23-25]:

$n(t)=C t^{-4} \exp \left[-2\left(t_{\mathrm{m}} / t\right)^{2}\right]$,

where $n(t)$ denotes the density of the desorbed dimers at time $t$ in the ionization region, and $t_{\mathrm{m}}=\ell(m / 2 k T)^{1 / 2}$ is the flight which corresponds to the maximum of the distribution. $C$ denotes a constant including experimental parameters such as the distance $\ell$ between the surface and the ionization region. The translational temperature $T$ of the $\mathrm{Na}$ dimers derived from the fit (Fig. 1) is $500 \mathrm{~K}$. For larger laser fluences (regime III), the experimental data can no longer be fitted by Eq. (1). Desorption in this regime is accompanied by a change of the shape of the time-of-flight spectra, since collisions of the desorbed species with each other in front of the surface come into effect. For completeness, it should be noted that non-thermal desorption can also be identified in the low fluence regime with $\phi<10 \mathrm{~mJ} \mathrm{~cm}^{-2}$. 
The dependence of the integral desorption signal (Fig. 2) shows two regimes in which the signal increases. They are separated by the above-mentioned plateau with a constant number of desorbed dimers. This dependence is quite unusual and differs markedly from the well-known vapor pressure curves with their exponential increase. It can be interpreted as follows. At low laser fluences, desorption occurs from binding sites of the rough $\mathrm{Na}$ surface which are characterized by a rather small energy necessary for bond breaking. At this stage the temperature rise needed to stimulate desorption (regime I, Fig. 2) can be quite low. The amount of desorbed $\mathrm{Na}$ dimers grows until the reservoir of these special sites is depleted. As a consequence, further increase of the fluence (and the temperature) does not give rise to a larger integral desorption signal. Instead, a plateau is reached. This interpretation is corroborated by identical maximum numbers of desorbed dimers, i.e. the same position of the plateau at about $4.5 \times 10^{4}$ registered $\mathrm{Na}_{2}^{+}$ions, irrespective of the laser wavelength applied to stimulate desorption. The size of the reservoir of the special sites alone determines the number of dimers which can be detached from the surface. Variation of the wavelength only results in different fluence values necessary to exhaust the reservoir. This reflects the different absorption cross-sections for $\hat{i}=532 \mathrm{~nm}$ and $\lambda=1064 \mathrm{~nm}$.

In a more general picture, the sites of low coordination number and small binding energy from which the dimers are detached can be regarded as defects of an ideal, low-index singlecrystal surface. Indeed, it has been shown in a variety of thermal and non-thermal desorption experiments on semiconductors, insulators and metals $[1,2,9,19]$ that such defects play a decisive role. If their number is reduced by annealing, the desorption rate for a given temperature rise should drop off sharply [9]. Furthermore, the plateau (regime II) must be expected to disappear. Comparison of trace a with traces b and $c$ in Fig. 3 supports this interpretation. After annealing, a much smaller desorption signal is observed and the plateau does no longer occurs. The number of desorbed dimers increases exponentially (traces $b$ and c), as expected for the classical dependerice of the vapor pressure on the substrate temperal ure.

If the laser fluence exceeds about $70 \mathrm{~mJ} \mathrm{~cm}^{-2}$ for $\lambda=1064 \mathrm{~nm}$ and $25 \mathrm{~mJ} \mathrm{~cm}^{-2}$ for $\lambda=53 . \mathrm{nm}$, the desorption rate increases further (regime III, Fig. 2). This can be explained by the desorption of dimers from a second kind of site with arger binding energy than those already depleted. Finally, for very high fluences, the integral desorption rate is limited by the $\mathrm{Na}$ coverage and saturates. The second kind of binding site can also be annealed (Fig. 3, trace c), but only at a temperature of $T=200 \mathrm{~K}$ as compared to the temperature $T=140 \mathrm{~K}$ needed to suppress desorption from the first site. After annealing at the higher temperature, thermal desorption is detectable only above $\phi=30 \mathrm{~mJ} \mathrm{~cm}^{-2}$ and then follows an exponential increase.

At present, the sites responsible for desorption cannot be identified and their electronic properties remain unknown. Possible sites could be dimers located at steps, kinks or on terraces. Measurements with different polarization of the laser light provide some hints from where the dimers might desorb and which type of electronic excitation could precede the rupture of the surface bond. For example, p-polarized radiation with $i=$ $532 \mathrm{~nm}$ stimulates desorption more efficiently as compared to s-polarized light. This indicates that p-polarized radiation predominantly interacts with a dipole in the direction normal to the surface of the oblate clusters. It therefore appears that $\mathrm{Na}_{2}$ is detached from terraces of the particle surface. Details will be published elsewhere [26]. The observation of two distinct binding sites from which the dimers desorb further indicates that the surface of the metal particles cannot be regarded as "amorphous", in the sense that arbitrary positions of the atoms with respect to each other and a wideranging continuous spectrum of binding energies would be allowed.

As already mentioned, the number of sodium dimers removed only amounts to $510 \%$ of the rate of desorbed monomers in a classical thermal desorption experiment (TPD) with a low heating rate $\left(10 \mathrm{~K} \mathrm{~s}^{-1}\right)$ [11]. This can be understood as follows: before the temperature where the evaporation of the particles in TPD commences is reached. 
the sites responsible for laser-induced thermal desorption have disappeared because of annealing. On the other hand, laser heating with elevated temperatures only persisting for at most several $\mu \mathrm{s}$ obviously does not last long enough for mass transport by diffusion and annealing to occur. This gives rise to the unusual observation that the composition of the desorption products depends upon the heating rate.

In conclusion, the results presented here show that specific binding sites at a metal surface not only play an essential role in non-thermal desorption $[9,11]$, but also in laser-induced thermal desorption. Due to the different desorption behavior of these sites, well-controlled smoothing of rough metal surfaces should be possible. In future experiments the latter issue will be investigated in more detail for different metals by direct imaging of the surface with scanning tunneling microscopy.

\section{Acknowledgements}

Financial support of the Fonds der Chemischen Industrie (F.T.) and the BMBF under grant number 13N 61597 (M.S.) is gratefully acknowledged.

\section{References}

[1] K. Ishikawa, J. Kanasaki, Y. Nakai, N. Itoh, Surf. Sci. 349 (1996) 153.

[2] H.T. Liu, Z. Wu, Phys. Rev. Lett. 72 (1994) 3891.

[3] W. Hoheisel, M. Vollmer, F. Träger, Phys. Rev. B 48 (1993) 17463.
[4] W. Hoheisel, K. Jungmann, M. Vollmer, R. Weidenauer, F. Träger, Phys. Rev. Lett. 60 (1988) 1649.

[5] M.J. Shea, R.N. Compton, Phys. Rev. B 47 (1993) 9967.

[6] I. Lee, J.E. Parks, II, T.A. Callcott, E.T. Arakawa, Phys. Rev. B 39 (1989) 8012.

[7] A.M. Bonch-Bruevich, T.A. Vartanyan, Yu.N. Maksimov, S.G. Przhibel'skii, V.V. Khromov, Sov. Phys. JETP 70 (1990) 993.

[8] A.M. Bonch-Bruevich, T.A. Vartanyan, Yu.N. Maksimov, S.G. Przhibel'skii, V.V. Khromov, Surf. Sci. 307-309 (1994) 350.

[9] T. Götz, M. Bergt, W. Hoheisel, F. Träger, M. Stuke, Appl. Phys. A 63 (1996) 315.

[10] M. Schildbach, A.V. Hamza, Phys. Rev. B 45 (1992) 6197.

[11] T. Götz, M. Bergt, W. Hoheisel, F. Träger, M. Stuke, Appl. Surf. Sci. 96-98 (1996) 280.

[12] J. Viereck, M. Stuke, F. Träger, Appl. Phys. A 64 (1997) 149.

[13] P.D. Brewer, M. Späth, M. Stuke, Mater. Res. Soc. Symp. Proc. 334 (1994) 245.

[14] R.W. Dreyfus, Appl. Phys. A 55 (1992) 335.

[15] D. Menzel, R. Gomer, J. Chem. Phys. 41 (1964) 3311.

[16] P.A. Redhead, Can. J. Phys. 42 (1964) 886.

[17] J.T. Dickinson, J.J. Shin, S.C. Langford, Appl. Surf. Sci. 96-98 (1996) 326.

[18] J.T. Dickinson, J.J. Shin, S.C. Langford, Appl. Surf. Sci. 96-98 (1996) 316.

[19] T. Gilbert, B. Dubreuil, M.F. Barthe, J.L. Debrun, J. Appl. Phys. 74 (1993) 3506.

[20] A. Hermann, E. Schumacher, L. Wöste, J. Chem. Phys. 68 (1978) 2327.

[21] T.D. Bennet, D.J. Krainovich, C.P. Grigoropoulos, Phys. Rev. Lett. 76 (1995) 1659.

[22] U. Kreibig, M. Vollmer, Optical Properties of Metal Clusters, Springer Series in Materials Science, vol. 25, Springer, Berlin, 1995.

[23] E. Hasselbrink, in: H.-L. Dai, W. Ho (Eds.), Laser Spectroscopy and Photochemistry on Metal Surfaces, part II, Advanced Series in Physical Chemistry, World Scientific, Singapore, 1995, p. 685.

[24] G. Wedler, H. Ruhmann, Surf. Sci. 121 (1982) 464.

[25] R. Kelly, R.W. Dreyfus, Surf. Sci. 198 (1988) 263.

[26] J. Viereck, F. Stietz, M. Stuke, T. Wenzel, F. Träger, to be published. 